\title{
Triaxiality in the interacting boson model
}

\author{
B. Sorgunlu ${ }^{\mathrm{a}, \mathrm{b}}$ and P. Van Isacker ${ }^{\mathrm{a}}$ \\ ${ }^{a}$ Grand Accélérateur National d'Ions Lourds, CEA/DSM-CNRS/IN2P3, \\ BP 55027, F-14076 Caen Cedex 5, France \\ ${ }^{\mathrm{b}}$ Department of Physics, Istanbul University, Istanbul, Turkey
}

\begin{abstract}
The signature splitting of the $\gamma$-vibrational band of several $\mathrm{Ru}, \mathrm{Pd}, \mathrm{Xe}, \mathrm{Ba}$, Os and $\mathrm{Pt}$ isotopes is analyzed in the framework of the interacting boson model (IBM). The nuclei studied are close to the $\gamma$-unstable $\mathrm{SO}(6)$ limit of the IBM and have well-known $\gamma$ bands. It is shown that in most nuclei the signature splitting is better reproduced by the inclusion of a three-body interaction between the $d$ bosons. In none of the nuclei evidence for a stable, triaxial ground-state shape is found.
\end{abstract}

Key words: interacting boson model, triaxiality, cubic interactions PACS: 21.60.Ev, 21.60.Fw, 21.10.Re

\section{Introduction}

Quadrupole deformations of atomic nuclei can be of two types: $\beta$ deformations which preserve axial symmetry and $\gamma$ deformations which lead to triaxial shapes. Although the collective model of nuclei introduced these ideas more than 50 years ago [1], it is still a matter of debate to what extent triaxiality is present in nuclei and, specifically, whether nuclear ground states exhibit stable triaxial deformation. On the other hand, $\gamma$ bands, associated with collective vibrations that break axial symmetry, are a well-established feature in the spectroscopy of deformed nuclei.

In this paper we undertake a detailed analysis of $\gamma$-band properties, in particular their signature splitting or even-odd staggering, in order to shed light on the question of triaxiality. The gist of the argument for doing this is as follows. Theoretical spectra derived from potentials which are either soft or rigid in the $\gamma$ degree of freedom, display $\gamma$ bands with different signature splitting. The observed energies of the $\gamma$-band members can therefore be used to fix the degree of softness or rigidity in $\gamma$. This idea is worked out here in the context of 
the interacting boson model (IBM) [2] which proposes an algebraic or grouptheoretical description of collective quadrupole excitations in nuclei. The IBM is particularly well suited for our present purpose for two reasons. First, its phenomenological application generally leads to accurate calculations of nuclear properties at low energy. As will be shown, a very precise description of the $\gamma$-band signature splitting can be obtained with three-body interactions between the bosons. Second, once the algebraic hamiltonian is fitted to the data, an intuitive, geometric picture in terms of $\beta$ and $\gamma$ deformations can be obtained in the so-called classical limit. In this way we establish a unbiased procedure which gauges the importance of triaxiality from the data.

In the present study we focus our attention on nuclei that are usually interpreted as soft in $\gamma$ [or close to the $\mathrm{SO}(6)$ limit of the IBM] and we investigate to what extent the observed signature splitting in the $\gamma$ band signals the occurrence of more rigid triaxiality.

\section{The interacting boson model}

In this section we give a brief description of the IBM with particular emphasis on the version of the model which includes higher-order interactions between the bosons. A full account of the IBM is given in ref. [2].

\subsection{The general hamiltonian}

The building blocks of the IBM are $s$ and $d$ bosons with angular momenta $\ell=0$ and $\ell=2$. A nucleus is characterized by a constant total number of bosons $N$ which equals half the number of valence nucleons (particles or holes, whichever is smaller). In this paper no distinction is made between neutron and proton bosons, an approximation which is known as IBM-1.

Since the hamiltonian of the IBM-1 conserves the total number of bosons, it

can be written in terms of the 36 operators $b_{\ell m}^{\dagger} b_{\ell^{\prime} m^{\prime}}$ where $b_{\ell m}^{\dagger}\left(b_{\ell m}\right)$ creates (annihilates) a boson with angular momentum $\ell$ and $z$ projection $m$. It can be shown [2] that this set of 36 operators generates the Lie algebra $U(6)$ of unitary transformations in six dimensions. A hamiltonian that conserves the total number of bosons is of the generic form

$$
\hat{H}=E_{0}+\hat{H}^{(1)}+\hat{H}^{(2)}+\hat{H}^{(3)}+\cdots,
$$

where the index refers to the order of the interaction in the generators of $U(6)$. The first term $E_{0}$ is a constant which represents the binding energy of the core. 
The second term is the one-body part

$$
\hat{H}^{(1)}=\epsilon_{s}\left[s^{\dagger} \times \tilde{s}\right]^{(0)}+\epsilon_{d} \sqrt{5}\left[d^{\dagger} \times \tilde{d}\right]^{(0)} \equiv \epsilon_{s} \hat{n}_{s}+\epsilon_{d} \hat{n}_{d}
$$

where $\times$ refers to coupling in angular momentum (shown as an upperscript in round brackets), $\tilde{b}_{\ell m} \equiv(-)^{\ell-m} b_{\ell,-m}$ and the coefficients $\epsilon_{s}$ and $\epsilon_{d}$ are the energies of the $s$ and $d$ bosons. The third term in the hamiltonian (1) represents the two-body interaction

$$
\hat{H}^{(2)}=\sum_{\ell_{1} \leq \ell_{2}, \ell_{1}^{\prime} \leq \ell_{2}^{\prime}, L} \tilde{v}_{\ell_{1} \ell_{2} \ell_{1}^{\prime} \ell_{2}^{\prime}}^{[}\left[\left[b_{\ell_{1}}^{\dagger} \times b_{\ell_{2}}^{\dagger}\right]^{(L)} \times\left[\tilde{b}_{\ell_{2}^{\prime}} \times \tilde{b}_{\ell_{1}^{\prime}}\right]^{(L)}\right]_{0}^{(0)},
$$

where the coefficients $\tilde{v}$ are related to the interaction matrix elements between normalized two-boson states,

$$
\left\langle\ell_{1} \ell_{2} ; L M\left|\hat{H}^{(2)}\right| \ell_{1}^{\prime} \ell_{2}^{\prime} ; L M\right\rangle=\sqrt{\frac{\left(1+\delta_{\ell_{1} \ell_{2}}\right)\left(1+\delta_{\left.\ell_{1}^{\prime} \ell_{2}^{\prime}\right)}\right.}{2 L+1}} \tilde{v}_{\ell_{1} \ell_{2} \ell_{1}^{\prime} \ell_{2}^{\prime}} .
$$

Since the bosons are necessarily symmetrically coupled, allowed two-boson states are $s^{2}(L=0), s d(L=2)$ and $d^{2}(L=0,2,4)$. Since for $n$ states with a given angular momentum one has $n(n+1) / 2$ interactions, seven independent two-body interactions $v$ are found: three for $L=0$, three for $L=2$ and one for $L=4$.

This analysis can be extended to higher-order interactions. One may consider, for example, the three-body interactions $\left\langle\ell_{1} \ell_{2} \ell_{3} ; L M\left|\hat{H}^{(3)}\right| \ell_{1}^{\prime} \ell_{2}^{\prime} \ell_{3}^{\prime} ; L M\right\rangle$. The allowed three-boson states are $s^{3}(L=0), s^{2} d(L=2), s d^{2}(L=0,2,4)$ and $d^{3}(L=0,2,3,4,6)$, leading to $6+6+1+3+1=17$ independent three-body interactions for $L=0,2,3,4,6$, respectively. Note that any three-boson state $s^{i} d^{3-i}$ is fully characterized by its angular momentum $L$; this is no longer the case for higher boson numbers when additional labels must be introduced.

The number of possible interactions at each order $n$ is summarized in table 1 for up to $n=3$. Some of these interactions exclusively contribute to the binding energy and do not influence the excitation spectrum of a nucleus. To determine the number of such interactions, one notes that the hamiltonian $\hat{N} \hat{H}^{(n-1)}$ for constant boson number (i.e., a single nucleus) essentially reduces to the $(n-1)$-body hamiltonian $\hat{H}^{(n-1)}$. Consequently, of the $N_{n}$ independent interactions of order $n$ contained in $\hat{H}^{(n)}, N_{n-1}$ terms of the type $\hat{N} \hat{H}^{(n-1)}$ must be discarded if one wishes to retain only those that influence the excitation energies. For example, given that there is one term of order zero (i.e., a constant), one of the two first-order terms (i.e., the combination $\hat{N}$ ) does not influence the excitation spectrum. Likewise, there are two first-order terms 
Table 1

Enumeration of $n$-body interactions in IBM-1 for $n \leq 3$.

\begin{tabular}{lrrr}
\hline Order & \multicolumn{3}{c}{ Number of interactions } \\
\cline { 2 - 4 } & total & type $\mathrm{I}^{a}$ & ${\text { type } \mathrm{II}^{b}}^{\text {toy }}$ \\
\hline$n=0$ & 1 & 1 & 0 \\
$n=1$ & 2 & 1 & 1 \\
$n=2$ & 7 & 2 & 5 \\
$n=3$ & 17 & 7 & 10 \\
\hline
\end{tabular}

${ }^{a}$ Interaction energy is constant for all states with the same $N$.

${ }^{b}$ Interaction energy varies from state to state.

(i.e., $\hat{n}_{s}$ and $\hat{n}_{d}$ ) and hence two of the seven two-body interactions do not influence the excitation spectrum. This argument leads to the numbers quoted in table 1.

We conclude that, in the nucleus-by-nucleus fits that will be performed in this work, there is a single one-boson energy of relevance, as well as five two-body and ten three-body interactions. This number of independent terms is too high for practical applications and simplifications must be sought on the basis of physical, empirical or formal arguments. Some of them are based on the classical limit of the IBM-1 to which we now turn.

\subsection{The classical limit}

The coherent-state formalism $[3,4,5]$ represents a bridge between algebraic and geometric nuclear models. The central outcome of the formalism is that for any IBM-1 hamiltonian a corresponding potential $V(\beta, \gamma)$ can be constructed where $\beta$ and $\gamma$ parametrize the intrinsic quadrupole deformation of the nucleus [6]. This procedure is known as the classical limit of the IBM-1.

The coherent states used for obtaining the classical limit of the IBM-1 are of the form

$$
\left|N ; \alpha_{\mu}\right\rangle \propto\left(s^{\dagger}+\sum_{\mu} \alpha_{\mu} d_{\mu}^{\dagger}\right)^{N}|\mathrm{o}\rangle,
$$

where $|\mathrm{o}\rangle$ is the boson vacuum and $\alpha_{\mu}$ are five complex variables. These have the interpretation of (quadrupole) shape variables and their associated conjugate momenta. If one limits oneself to static problems, the $\alpha_{\mu}$ can be taken as real; they specify a shape and are analogous to the shape variables of the 
droplet model of the nucleus [6]. The $\alpha_{\mu}$ can be related to three Euler angles which define the orientation of an intrinsic frame of reference, and two intrinsic shape variables, $\beta$ and $\gamma$, that parametrize quadrupole vibrations of the nuclear surface around an equilibrium shape. In terms of the latter variables, the coherent state (5) is rewritten as

$$
|N ; \beta \gamma\rangle \propto\left(s^{\dagger}+\beta\left[\cos \gamma d_{0}^{\dagger}+\sqrt{\frac{1}{2}} \sin \gamma\left(d_{-2}^{\dagger}+d_{+2}^{\dagger}\right)\right]\right)^{N}|\mathrm{o}\rangle .
$$

The expectation value of the hamiltonian (1) in this state can be determined by elementary methods [7] and yields a function of $\beta$ and $\gamma$ which is identified with a potential $V(\beta, \gamma)$, familiar from the geometric model. In this way the following classical limit of the hamiltonian (1) is found:

$$
V(\beta, \gamma)=E_{0}+\sum_{n \geq 1} \frac{N(N-1) \cdots(N-n+1)}{\left(1+\beta^{2}\right)^{n}} \sum_{k l} a_{k l}^{(n)} \beta^{2 k+3 l} \cos ^{l} 3 \gamma
$$

where the non-zero coefficients $a_{k l}^{(n)}$ of order $n=1,2$ and 3 are given by

$$
\begin{aligned}
& a_{00}^{(1)}=\epsilon_{s}, \quad a_{10}^{(1)}=\epsilon_{d}, \\
& a_{00}^{(2)}=\frac{1}{2} v_{s s s s}^{0}, \quad a_{10}^{(2)}=\sqrt{\frac{1}{5}} v_{s s d d}^{0}+v_{s d s d}^{2}, \quad a_{01}^{(2)}=-\frac{2}{\sqrt{7}} v_{s d d d}^{2}, \\
& a_{20}^{(2)}=\frac{1}{10} v_{d d d d}^{0}+\frac{1}{7} v_{d d d d}^{2}+\frac{9}{35} v_{d d d d}^{4}, \\
& a_{00}^{(3)}=\frac{1}{6} v_{s s s s s s}^{0}, \quad a_{10}^{(3)}=\sqrt{\frac{1}{15}} v_{s s s s d d}^{0}+\frac{1}{2} v_{s s d s s d}^{2} \\
& a_{01}^{(3)}=-\frac{1}{3} \sqrt{\frac{2}{35}} v_{s s s d d d}^{0}-\sqrt{\frac{2}{7}} v_{s s d s d d}^{2}, \\
& a_{20}^{(3)}=\frac{1}{10} v_{s d d s d d}^{0}+\sqrt{\frac{1}{7}} v_{s s d d d d}^{2}+\frac{1}{7} v_{s d d s d d}^{2}+\frac{9}{35} v_{s d d s d d}^{4} \\
& a_{11}^{(3)}=-\frac{1}{5} \sqrt{\frac{2}{21}} v_{s d d d d d}^{0}-\frac{\sqrt{2}}{7} v_{s d d d d d}^{2}-\frac{18}{35} \sqrt{\frac{2}{11}} v_{s d d d d d}^{4} \\
& a_{30}^{(3)}=\frac{1}{14} v_{d d d d d d}^{2}+\frac{1}{30} v_{d d d d d d}^{3}+\frac{3}{154} v_{d d d d d d}^{4}+\frac{7}{165} v_{d d d d d d}^{6} \\
& a_{02}^{(3)}=\frac{1}{105} v_{d d d d d d}^{0}-\frac{1}{30} v_{d d d d d d}^{3}+\frac{3}{110} v_{d d d d d d}^{4}-\frac{4}{1155} v_{d d d d d d}^{6}
\end{aligned}
$$

in terms of the single boson energies $\epsilon_{s}$ and $\epsilon_{d}$, and the matrix elements between normalized two- and three-body states, 


$$
\begin{aligned}
v_{\ell_{1} \ell_{2} \ell_{1}^{\prime} \ell_{2}^{\prime}}^{L} & =\left\langle\ell_{1} \ell_{2} ; L M\left|\hat{H}^{(2)}\right| \ell_{1}^{\prime} \ell_{2}^{\prime} ; L M\right\rangle, \\
v_{\ell_{1} \ell_{2} \ell_{3} \ell_{1}^{\prime} \ell_{2}^{\prime} \ell_{3}^{\prime}} & =\left\langle\ell_{1} \ell_{2} \ell_{3} ; L M\left|\hat{H}^{(3)}\right| \ell_{1}^{\prime} \ell_{2}^{\prime} \ell_{3}^{\prime} ; L M\right\rangle .
\end{aligned}
$$

The expressions (7) and (8) will be useful for making a choice between the many possible three-body interactions.

\subsection{A specific two-body hamiltonian}

From a great number of standard IBM-1 studies [2] one has a good idea of a workable hamiltonian with up to two-body interactions which is of the form

$$
\hat{H}^{(1+2)}=\epsilon_{d} \hat{n}_{d}+\kappa \hat{Q} \cdot \hat{Q}+\kappa^{\prime} \hat{L} \cdot \hat{L}+\lambda_{d} \hat{n}_{d}^{2}
$$

where $\hat{Q}$ is the quadrupole operator with components

$$
\hat{Q}_{\mu}=\left[d^{\dagger} \times \tilde{s}+s^{\dagger} \times \tilde{d}\right]_{\mu}^{(2)}+\chi\left[d^{\dagger} \times \tilde{d}\right]_{\mu}^{(2)},
$$

and $\hat{L}$ is the angular momentum operator, $\hat{L}_{\mu}=\sqrt{10}\left[d^{\dagger} \times \tilde{d}\right]_{\mu}^{(1)}$. The $\hat{Q}^{2}$ and $\hat{L}^{2}$ terms in (10) constitute the hamiltonian of the so-called consistent- $Q$ formalism (CQF) [8]. Its eigenfunctions are fully determined by $\chi$ which for $\chi= \pm \sqrt{7} / 2$ gives rise to the deformed or $\mathrm{SU}(3)$ limit and for $\chi=0$ to the $\gamma$-unstable or $\mathrm{SO}(6)$ limit. In an extended consistent- $Q$ formalism (ECQF) [9] a further term $\hat{n}_{d}$ is added with which the third, vibrational or $\mathrm{U}(5)$ limit of the IBM-1 can be obtained. The ECQF hamiltonian thus allows one to reach all three limits of the model with four parameters. In some nuclei an additional term $\lambda_{d} \hat{n}_{d}^{2}$ further improves the description of the excitation spectrum. The effect of this term with $\lambda_{d}<0$ is an increase of the moment of inertia with increasing angular momentum (or $d$-boson seniority $\tau$ ). This so-called ' $\tau$-compression' has been used for the first time in ref. [10].

For the calculation of electric quadrupole properties an E2 transition operator is needed. In the IBM-1 it is defined as $\hat{T}_{\mu}(\mathrm{E} 2)=e_{\mathrm{b}} \hat{Q}_{\mu}$ where $e_{\mathrm{b}}$ is an effective charge for the bosons. In CQF the quadrupole operator in the E2 operator and in the hamiltonian are the same [8], that is, they contain the same $\chi$.

\subsection{A specific three-body hamiltonian}

Many nuclear properties can be correctly described by the relatively simple hamiltonian (10) but some cannot. A notable example is the even-odd staggering in the $\gamma$ band of nuclei that are close to the $\mathrm{SO}(6)$ limit. A characteristic 
feature of the $\gamma$-unstable limit of the IBM- 1 is a bunching of $\gamma$-band states [11] according to $2^{+},\left(3^{+}, 4^{+}\right),\left(5^{+}, 6^{+}\right), \ldots$, that is, $3^{+}$and $4^{+}$are close in energy, etc. This even-odd staggering is observed in certain $\mathrm{SO}(6)$ nuclei but not in all and in some it is, in fact, replaced by the opposite bunching $\left(2^{+}, 3^{+}\right)$, $\left(4^{+}, 5^{+}\right), \ldots$, which is typical of a rigid triaxial rotor [12]. From these qualitative observations it is clear that the even-odd $\gamma$-band staggering is governed by the $\gamma$ degree of freedom (i.e., triaxiality) as it changes character in the transition from a $\gamma$-soft vibrator to a rigid triaxial rotor.

A proper description of triaxiality in the IBM-1 must necessarily involve higher-order interactions as can be shown from the expressions given in sect. 2.2. The minimum of the potential $V(\beta, \gamma)$ in (7) (which can be thought of as the equilibrium shape of the nucleus) of an IBM-1 hamiltonian with up to twobody interactions is either spherical $(\beta=0)$, prolate deformed $\left(\beta>0, \gamma=0^{\circ}\right)$ or oblate deformed $\left(\beta>0, \gamma=60^{\circ}\right)$. The lowest term in (7) with a triaxial extremum is quadratic in $\cos 3 \gamma(l=2)$ and this requires a non-zero $a_{02}^{(3)}$ coefficient. From the explicit expressions given in eqs. (8) it is seen that the lowest-order interactions possibly leading to a triaxial minimum in $V(\beta, \gamma)$ are thus necessarily of the form

$$
\hat{H}_{d}^{(3)}=\sum_{L} \tilde{v}_{d d d d d d}^{L}\left[\left[d^{\dagger} \times d^{\dagger}\right]^{(\lambda)} \times d^{\dagger}\right]^{(L)} \cdot\left[[\tilde{d} \times \tilde{d}]^{\left(\lambda^{\prime}\right)} \times \tilde{d}\right]^{(L)},
$$

where the allowed angular momenta are $L=0,2,3,4,6$. For several $L$ more than one combination of intermediate angular momenta $\lambda$ and $\lambda^{\prime}$ is possible; these do not give rise to independent terms but differ by a scale factor. To avoid the confusion caused by this scale factor, we rewrite the hamiltonian (12) as

$$
\hat{H}_{d}^{(3)}=\sum_{L} v_{d d d d d d}^{L} \hat{B}_{L}^{\dagger} \cdot \tilde{B}_{L}, \quad \hat{B}_{L M}^{\dagger}=N_{\lambda L}\left[\left[d^{\dagger} \times d^{\dagger}\right]^{(\lambda)} \times d^{\dagger}\right]_{M}^{(L)} .
$$

For simplicity's sake the coefficients $v_{d d d d d}^{L}$ shall be denoted as $v_{L}$ in the following. The normalization coefficient $N_{\lambda L}$ is defined such that $B_{L M}\left|d^{3} ; L M\right\rangle$ yields the vacuum state $|\mathrm{o}\rangle$, where $\left|d^{3} ; L M\right\rangle$ is a normalized, symmetric state of three bosons coupled to total angular momentum $L$ and $z$ projection $M$. The normalization coefficients $N_{\lambda L}$ are given in table 2 for the different combinations of $\lambda$ and $L$. Results are independent of $\lambda$ provided the appropriate coefficient $N_{\lambda L}$ is used.

While there are good arguments for choosing any of the three-body terms $\hat{B}_{L}^{\dagger} \cdot \tilde{B}_{L}$, it is more difficult to distinguish a priori between these five different interactions. From the expression for $a_{02}^{(3)}$ given in eqs. (8) it is seen that the cubic term $\hat{B}_{L}^{\dagger} \cdot \tilde{B}_{L}$ with $L=3$ is proportional to $\sin ^{2} 3 \gamma$. It is therefore the interaction which is most effective to create a triaxial minimum in the potential 
Table 2

Normalization coefficients $N_{\lambda L}$ for three-d-boson states.

\begin{tabular}{cccccc}
\hline$L$ & 0 & 2 & 3 & 4 & 6 \\
\hline$\lambda=0$ & - & $\sqrt{\frac{5}{14}}$ & - & - & - \\
$\lambda=2$ & $\sqrt{\frac{1}{6}}$ & $\sqrt{\frac{7}{8}}$ & $\sqrt{\frac{7}{30}}$ & $\sqrt{\frac{7}{22}}$ & - \\
$\lambda=4$ & - & $\sqrt{\frac{35}{72}}$ & $-\sqrt{\frac{7}{12}}$ & $\sqrt{\frac{7}{20}}$ & $\sqrt{\frac{1}{6}}$ \\
\hline
\end{tabular}

$V(\beta, \gamma)$ and for this reason it has been studied in most detail. The effect of $\hat{B}_{3}^{\dagger} \cdot \tilde{B}_{3}$ on even-odd staggering in the $\gamma$ band was demonstrated with numerical calculations [13]. Applications of the $L=3$ three-body term were proposed in ref. [14] for $\mathrm{SO}(6)$-like $\mathrm{Xe}$ and $\mathrm{Ba}$ isotopes in the mass region around $A=130$, as well as for ${ }^{196} \mathrm{Pt}$.

Besides these physical and empirical arguments, there are also attractive formal aspects of the $\hat{B}_{3}^{\dagger} \cdot \tilde{B}_{3}$ interaction among the $d$ bosons. A first one concerns its effect in the $\mathrm{SU}(3)$ limit of the IBM-1. In this limit states are characterized by the $\mathrm{U}(6)$ label $[N]$, the $\mathrm{SU}(3)$ labels $(\lambda, \mu)$, the angular momentum or $\mathrm{SO}(3)$ label $L$ and its $z$ projection $M$, and by an $\mathrm{SU}(3) \supset \mathrm{SO}(3)$ multiplicity label $\kappa$ [15]. Likewise, any interaction can be written in terms of products of tensor operators $\hat{T}_{[N](\lambda, \mu) \kappa L M}^{\dagger}$ [which creates an $N$-boson state with the quantum numbers $(\lambda, \mu) \kappa L M]$ and their hermitian conjugates. Since a three-boson state with $L=3$ is unique, it follows that its $\mathrm{SU}(3)$ labels are fixed, namely, $(\lambda, \mu)=(2,2)$ and $\kappa=2$, and that the following proportionality must hold:

$$
B_{L=3, M}^{\dagger} \propto \hat{T}_{[3](2,2) \kappa=2, L=3, M}^{\dagger} .
$$

This property can be used to show that

$$
\begin{aligned}
& \tilde{B}_{L=3, M}\left|[N](2 N, 0) L^{\prime} M^{\prime}\right\rangle=0, \\
& \tilde{B}_{L=3, M}\left|[N](2 N-4,2) \kappa^{\prime}=0, L^{\prime} M^{\prime}\right\rangle=0,
\end{aligned}
$$

that is, the $\hat{B}_{3}^{\dagger} \cdot \tilde{B}_{3}$ interaction acting on the ground-state band $(2 N, 0)$ or on the $\beta$-vibrational band $(2 N-4,2) \kappa=0$ gives zero. The former property results from the fact that the $\mathrm{SU}(3)$ Kronecker product $(2 N, 0) \times(2,2)$ does not yield an $\mathrm{SU}(3)$ representation that is contained in the $\mathrm{U}(6)$ representation $[N-3]$.

The situation can be summarized by stating that the $\mathrm{SU}(3)$ hamiltonian augmented with a $\hat{B}_{3}^{\dagger} \cdot \tilde{B}_{3}$ interaction is an example of a partial dynamical symmetry [16]: while the eigenstates of this extended hamiltonian are not solvable in general, some of them are, in particular the members of the ground-state and $\beta$ bands. In first approximation the effect of $\hat{B}_{3}^{\dagger} \cdot \tilde{B}_{3}$ is to shift the entire $\gamma$ 


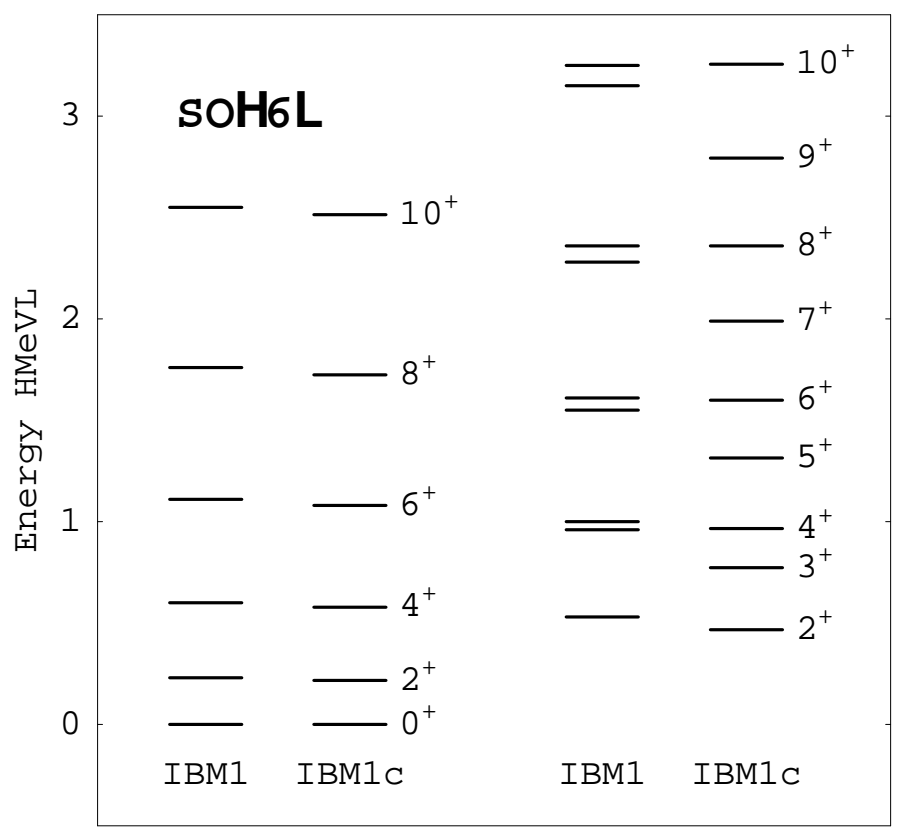

Fig. 1. Levels of the ground-state and $\gamma$ bands up to angular momentum $J^{\pi}=10^{+}$ in the exact $\mathrm{SO}(6)$ limit (labelled 'IBM1') and in the $\mathrm{SO}(6)$ limit plus the three-body interaction $\hat{B}_{3}^{\dagger} \cdot \tilde{B}_{3}$ (labelled 'IBM1c'). The IBM-1 hamiltonian (10) is used for $\chi=0$ with $\kappa=-50 \mathrm{keV}, \kappa^{\prime}=5 \mathrm{keV}$ and $\epsilon_{d}=\lambda_{d}=0$; the strength of the three-body term is $v_{3}=-50 \mathrm{keV}$. The number of bosons is $N=10$.

band in energy without changing its moment of inertia or the structure of its states. We also note that this nicely complements the (two-body) interaction derived previously [17] which leaves the ground-state and $\gamma$ bands solvable but modifies the structure of the $\beta$ band.

Let us now turn to the effect of the $\hat{B}_{3}^{\dagger} \cdot \tilde{B}_{3}$ interaction in the $\mathrm{SO}(6)$ limit of the IBM-1. Its influence on the energy spectrum is illustrated in fig. 1 . It is seen that the ground-state band levels are only slightly affected by the cubic interaction while the effect on the $\gamma$-band energies is important. In particular, the even-odd staggering in the $\gamma$ band, characteristic of $\gamma$-soft or $\mathrm{SO}(6)$ behaviour, is greatly diminished. A sensitive way of testing the signature splitting of the $\gamma$ band is through $S(J)$ given by [18]

$$
S(J)=\frac{E(J)-E(J-1)}{E(J)-E(J-2)} \cdot \frac{J(J+1)-(J-1)(J-2)}{J(J+1)-J(J-1)}-1,
$$

which vanishes if there is no even-odd staggering. This quantity is shown in fig. 2 for the schematic $\mathrm{SO}(6)$ case. The figure confirms that the three-body interaction $\hat{B}_{3}^{\dagger} \cdot \tilde{B}_{3}$ (with $v_{3}<0$ ) has the property of reducing the $\gamma$-band staggering but it also reveals that this reduction is more important for the high- $J$ levels. This is a characteristic feature that can be experimentally tested. 


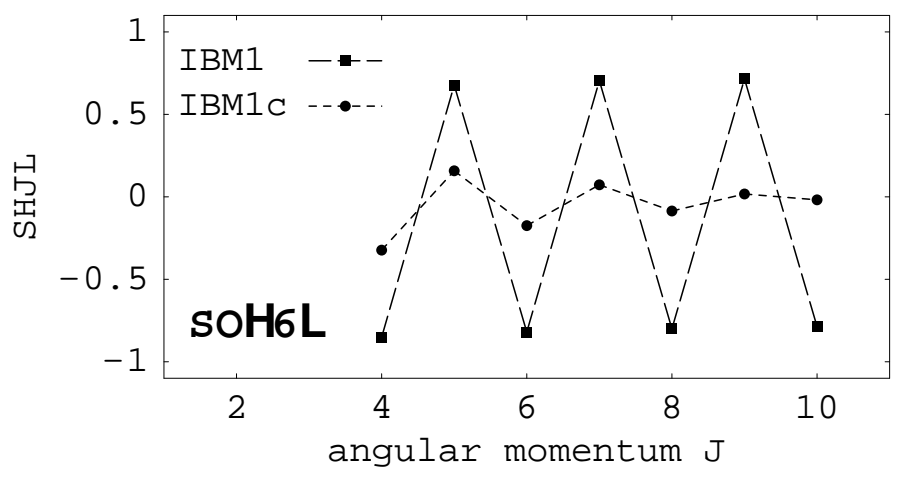

Fig. 2. The signature splitting of the $\gamma$ band in the exact $\mathrm{SO}(6)$ limit (labelled 'IBM1') and in the $\mathrm{SO}(6)$ limit plus the three-body interaction $\hat{B}_{3}^{\dagger} \cdot \tilde{B}_{3}$ (labelled 'IBM1c'). The parameters of the hamiltonian are given in the caption of fig. 1.
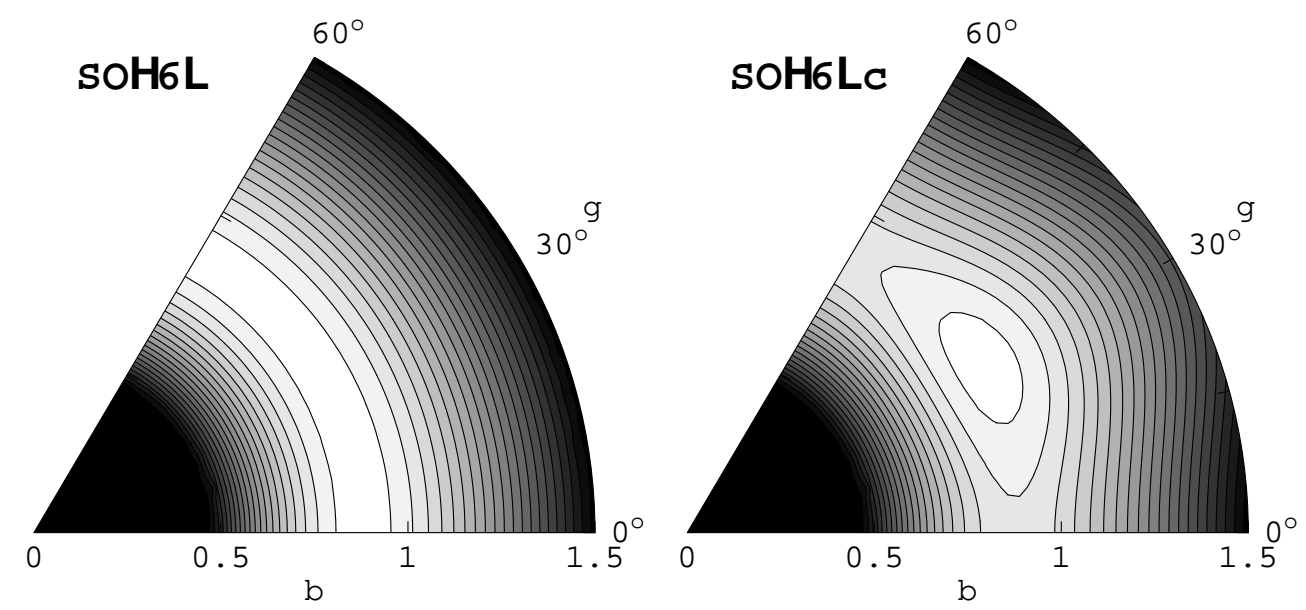

Fig. 3. Potential energy surfaces $V(\beta, \gamma)$ in the $\mathrm{SO}(6)$ limit. The plot on the left-hand side shows the classical limit of the IBM-1 hamiltonian in the $\mathrm{SO}(6)$ limit while on the right-hand side the effect of $\hat{B}_{3}^{\dagger} \cdot \tilde{B}_{3}$ is included. The parameters of the hamiltonian are given in the caption of fig. 1.

Figure 3 illustrates the effect of the cubic interaction $\hat{B}_{3}^{\dagger} \cdot \tilde{B}_{3}$ on the potential $V(\beta, \gamma)$ derived in the classical limit. While the potential in the $\mathrm{SO}(6)$ limit is completely $\gamma$ independent, a shallow triaxial minimum develops for $v_{3}<0$. We note, however, that this triaxial minimum exists precariously for $\chi=0$ and quickly disappears when $\chi$ acquires non-zero values.

Most of the results presented below are obtained with the $d$-boson cubic interaction with $L=3$ which in general reproduces best the $\gamma$-band properties. We have nevertheless systematically investigated the terms with $L \neq 3$, and will occasionally refer to those results in the following. 


\section{Numerical procedure}

To test effectiveness of the various cubic interactions in reproducing the data in near-SO(6) nuclei, we have devised the following fitting procedure. The nuclei considered should have enough known states in the ground-state and $\gamma$ bands - preferably up to angular momentum $J^{\pi}=10^{+}$- for the procedure to be meaningful. The first step is to determine the parameters in the standard IBM-1 hamiltonian (10). For an initial choice of $\chi$, the parameters $\kappa$ and $\kappa^{\prime}$ are first determined while keeping $\epsilon_{d}$ and $\lambda_{d}$ zero. With $\left(\kappa, \kappa^{\prime}\right)$ thus found as starting values, a new fit is performed setting $\epsilon_{d}$ free as well, leading to the best values $\left(\kappa, \kappa^{\prime}, \epsilon_{d}\right)$. Finally, this process is repeated by letting also $\lambda_{d}$ free, leading to a final set $\left(\kappa, \kappa^{\prime}, \epsilon_{d}, \lambda_{d}\right)$ for a given $\chi$. In some nuclei, especially if not enough data are available, the last step can prove numerically unstable and no unique set $\left(\kappa, \kappa^{\prime}, \epsilon_{d}, \lambda_{d}\right)$ is found. In that case we leave $\lambda_{d}$ equal to zero. The parameter $\chi$ cannot be reliably determined from energies but is fixed from E2 transition rates which are calculated in CQF. If not enough E2 data are available, we take $\chi$ from a neighbouring isotope. The entire procedure is repeated for different $\chi$, retaining the value that gives the best agreement with

the E2 data. In a last step the importance of the $\hat{B}_{L}^{\dagger} \cdot \tilde{B}_{L}$ terms is tested in a similar way by allowing the variation of all five parameters $\left(\kappa, \kappa^{\prime}, \epsilon_{d}, \lambda_{d}, v_{L}\right)$ - or four if $\lambda_{d}=0$-while keeping $\chi$ constant. Since we are particularly interested in the influence of $v_{L}$ on the even-odd staggering, in this final step we adjust this parameter while assigning a larger weight to the members of the $\gamma$ band. The accuracy of the fits can be tested by plotting the signature splitting $S(J)$ which will be done systematically.

\section{Results and discussion}

In this section we present the results for 16 different nuclei ranging from neutron-rich $\mathrm{Ru}$ and $\mathrm{Pd}$ isotopes, via $\mathrm{Xe}$ and $\mathrm{Ba}$ nuclei with mass number $A \sim 130$ to neutron-deficient $\mathrm{Os}$ and $\mathrm{Pt}$ isotopes. All nuclei are close to the $\mathrm{SO}(6)$ limit of the IBM-1 and have $\gamma$ bands that are known at least up to $J^{\pi}=7^{+}$. Since the IBM-1 is a low-spin model, states up to $J^{\pi}=10^{+}$but not higher are included in the fits. All data have been retrieved from the Brookhaven National Nuclear Data Center [19] unless indicated otherwise.

\subsection{Ruthenium and palladium isotopes}

In the context of the interacting boson model, the $\mathrm{Ru}$ and $\mathrm{Pd}$ isotopes are described as transitional between vibrational [or $\mathrm{U}(5)]$ and $\gamma$ soft [or $\mathrm{SO}(6)]$ [20]. 
Table 3

Parameters and rms deviation for $\mathrm{Ru}$ and $\mathrm{Pd}$ isotopes in units of $\mathrm{keV}$.

\begin{tabular}{cccccccc}
\hline Nucleus & $\epsilon_{d}$ & \multicolumn{1}{c}{$\kappa$} & $\kappa^{\prime}$ & \multicolumn{1}{c}{$\lambda_{d}$} & $v_{3}$ & $\chi^{*}$ & $\sigma$ \\
\hline${ }^{108} \mathrm{Ru}$ & 1078 & -57.6 & 12.1 & -144.9 & - & -0.10 & 23 \\
& 852 & -66.8 & 8.3 & -130.7 & -13.1 & -0.10 & 45 \\
& 732 & -74.6 & 14.0 & -157.8 & $30.5^{* *}$ & -0.10 & 19 \\
${ }^{110} \mathrm{Ru}$ & 1053 & -46.1 & 15.5 & -123.7 & - & -0.10 & 39 \\
& 873 & -56.9 & 9.9 & -108.5 & -28.1 & -0.10 & 20 \\
${ }^{112} \mathrm{Ru}$ & 837 & -45.3 & 15.2 & -116.8 & - & -0.10 & 55 \\
& 424 & -57.8 & 7.7 & -73.7 & -46.8 & -0.10 & 38 \\
\hline${ }^{114} \mathrm{Pd}$ & 321 & 1.0 & 12.2 & - & - & -0.10 & 91 \\
& 486 & -19.6 & 5.7 & - & -94.6 & -0.10 & 61 \\
${ }^{116} \mathrm{Pd}$ & 367 & -3.5 & 12.4 & - & - & -0.10 & 85 \\
& 451 & -19.1 & 8.3 & - & -84.3 & -0.10 & 56 \\
\hline
\end{tabular}

${ }^{*}$ Dimensionless. ${ }^{* *}$ Value of the coefficient $v_{2}$.

In this interpretation the neutron-rich members of these isotopic chains are close to the $\mathrm{SO}(6)$ limit of the IBM-1 and thus they fall into the class of nuclei we wish to study in this work.

Recently, gamma-ray spectroscopy of the fission fragments produced by a ${ }^{252} \mathrm{Cf}$ source has significantly improved our knowledge of the structure of the isotopes ${ }^{108-112} \mathrm{Ru}[21]$. The new data that became available on these nuclei was considered in ref. [22] where particular attention was paid to the staggering pattern in the $\gamma$ band and the triaxial degree of freedom. The advantage of the method as described in sect. 3 is that a consistent one- and two-body IBM-1 hamiltonian is taken to which a three-body term is added without changing the value of $\chi$. In this way any improvement of the description of the $\gamma$-band staggering can be unambiguously attributed to the three-body term. Also, a least-squares fit is performed to the parameters in the hamiltonian according to the procedure outlined in sect. 3. In spite of these differences the results obtained here are globally in agreement with those of Stefanescu et al. [22]. The main conclusion is that, while the staggering pattern of the $\gamma$ band is much improved with the $\hat{B}_{3}^{\dagger} \cdot \tilde{B}_{3}$ interaction in ${ }^{110} \mathrm{Ru}$ and ${ }^{112} \mathrm{Ru}$, this is not the case for ${ }^{108} \mathrm{Ru}$. This is evident from the parameters shown in table 3 . The root-mean-square ( $\mathrm{rms}$ ) deviation $\sigma$ actually increases for ${ }^{108} \mathrm{Ru}$ when the three-body interaction is added to the hamiltonian as a consequence of the fit procedure which gives more weight to the $\gamma$-band members when also $v_{3}$ is fitted. The increase in $\sigma$ illustrates that the $\gamma$-band energies in ${ }^{108} \mathrm{Ru}$ 


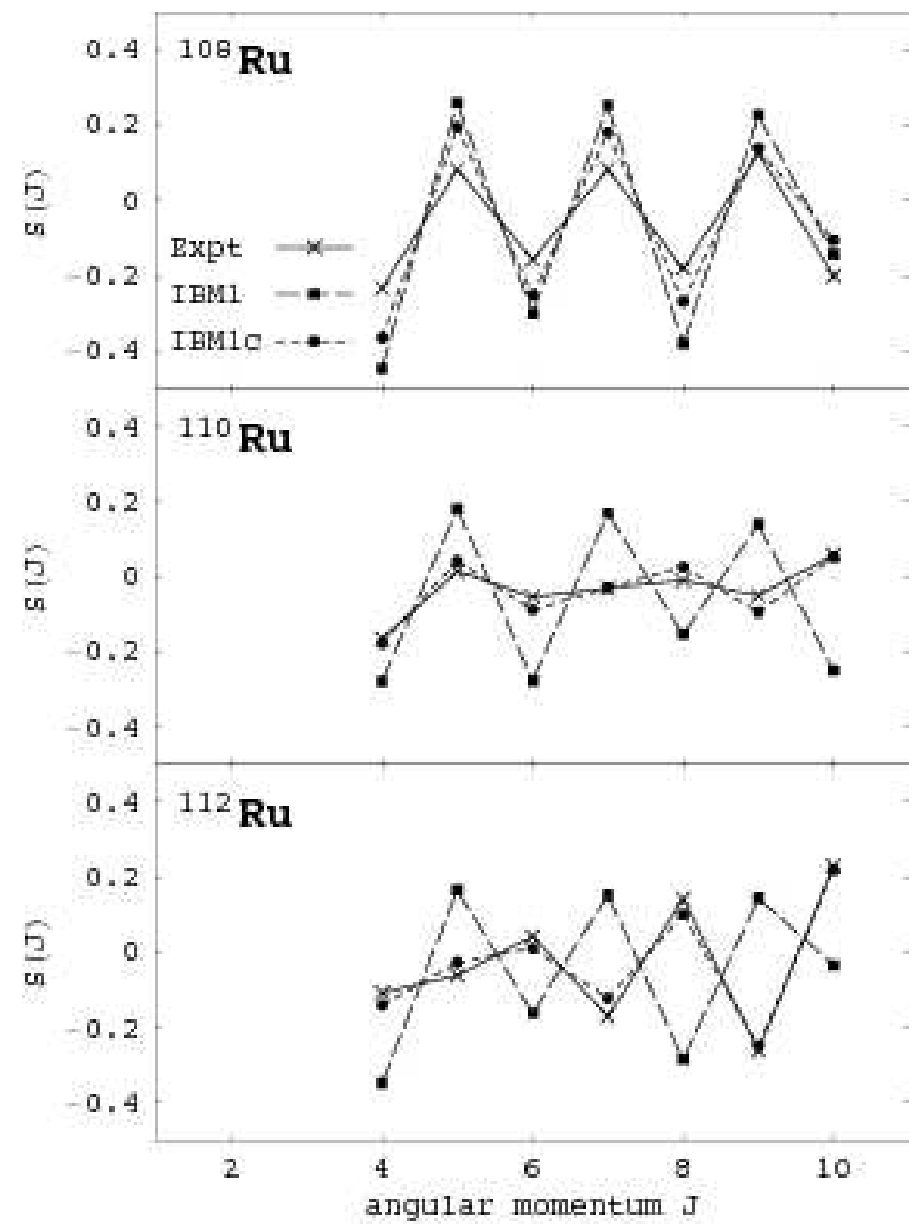

Fig. 4. Observed and calculated signature splitting of the $\gamma$ bands in ${ }^{108-112} \mathrm{Ru}$. The data are indicated by crosses and the results of the IBM-1 without and with the three-body interaction $\hat{B}_{3}^{\dagger} \cdot \tilde{B}_{3}$ by squares and dots, respectively.

cannot be reproduced by adding $\hat{B}_{3}^{\dagger} \cdot \tilde{B}_{3}$ without destroying the agreement for the ground-state band.

As one goes to the heavier $\mathrm{Ru}$ isotopes, one notices a distinct evolution of the even-odd staggering pattern (see fig. 4). Whereas the staggering pattern is essentially consistent with the IBM-1 calculation without cubic interactions in ${ }^{108} \mathrm{Ru}$, this is no longer the case in the two heavier isotopes. In ${ }^{110} \mathrm{Ru}$ there is very little staggering at all, $S(J) \approx 0$, and in ${ }^{112} \mathrm{Ru}$ the staggering pattern in the data is in fact the reverse of what is obtained without cubic interactions, especially at higher angular momenta. The $\hat{B}_{3}^{\dagger} \cdot \tilde{B}_{3}$ interaction shifts levels with even (odd) angular momentum upwards (downwards) in energy and it does so increasingly with increasing spin. This is exactly what can be observed from the data in ${ }^{110} \mathrm{Ru}$ and ${ }^{112} \mathrm{Ru}$ and this provides a strong phenomenological argument for the use of the $\hat{B}_{3}^{\dagger} \cdot \tilde{B}_{3}$ interaction. 


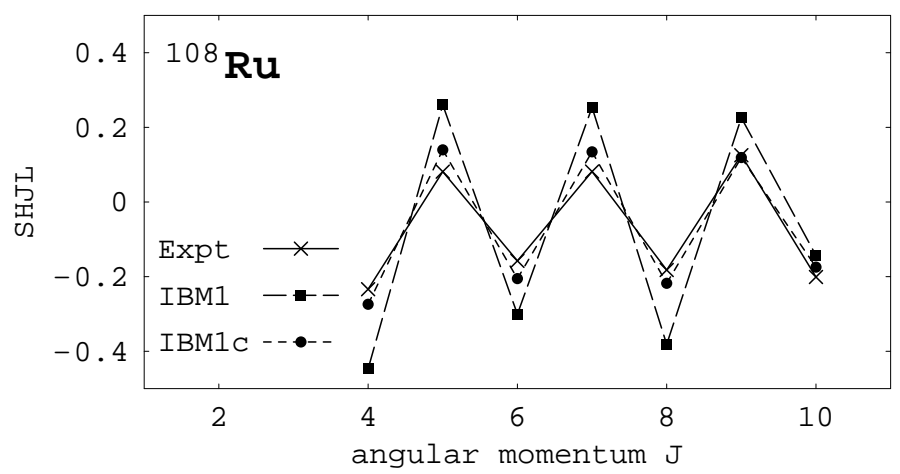

Fig. 5. Same caption as fig. 4 for the nucleus ${ }^{108} \mathrm{Ru}$ and the three-body interaction $\hat{B}_{2}^{\dagger} \cdot \tilde{B}_{2}$.

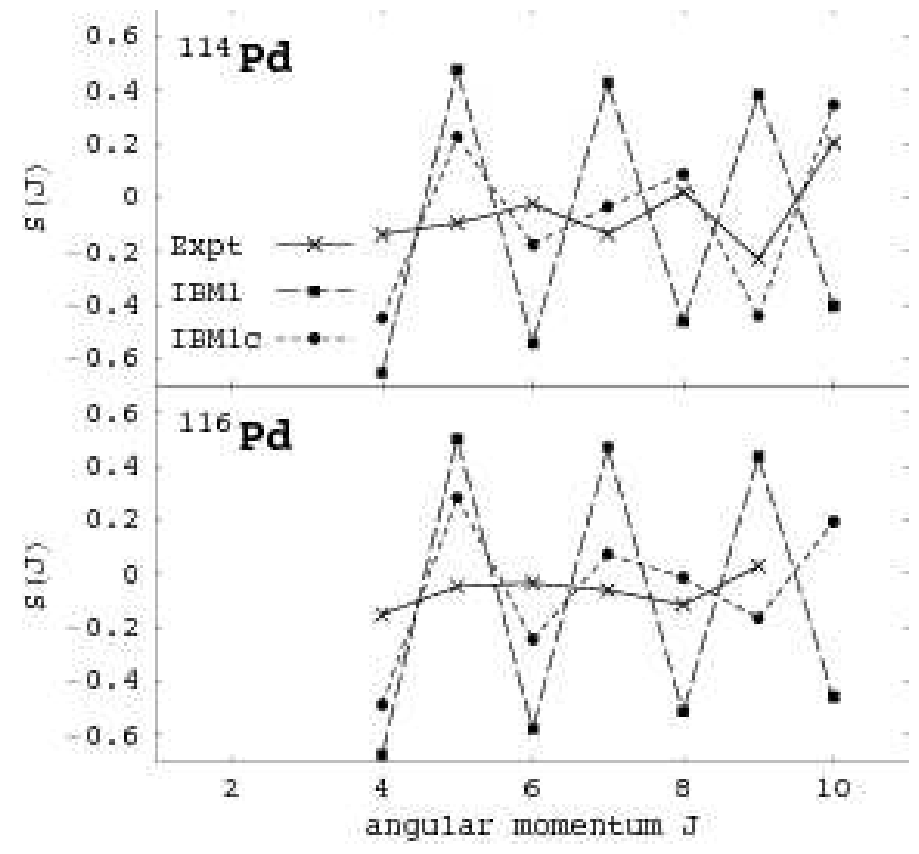

Fig. 6. Same caption as fig. 4 for the nuclei ${ }^{114,116} \mathrm{Pd}$.

From the plot of the signature splitting we can also 'understand' why the $\hat{B}_{3}^{\dagger} \cdot \tilde{B}_{3}$ interaction fails in ${ }^{108} \mathrm{Ru}$ : the deviations in staggering between the data and the IBM-1 calculation without cubic interactions actually decrease rather than increase with angular momentum. This feature is incompatible with the $L=3$ term in the hamiltonian (12) but is exactly what is obtained with the $L=2$ term as shown in fig. 5 .

The results for the $\gamma$-band staggering in the ${ }^{114,116} \mathrm{Pd}$ isotopes are shown in fig. 6. In these nuclei one cannot reliably determine the coefficient $\lambda_{d}$ of $\hat{n}_{d}^{2}$ which is therefore kept zero. The inclusion of the three-body interaction $\hat{B}_{3}^{\dagger} \cdot \tilde{B}_{3}$ does improve the fit substantially (see the rms deviations in table 3) but even with this term the observed staggering pattern cannot be reproduced.

It is important to check that the cubic hamiltonian thus obtained gives reason- 
able results as regards electric quadrupole transitions. This will be illustrated for some of the isotopes discussed in the subsequent subsections. For the Ru isotopes it has been shown to be the case in ref. [22] while the relevant E2 data are not known for ${ }^{114,116} \mathrm{Pd}$.

Once the parameters of the hamiltonian have been fitted to the energy spectrum and E2 transition rates, its classical limit yields a potential energy surface $V(\beta, \gamma)$ as obtained from the expression $(7)$. In this way it can be verified to what extent triaxial features are introduced by the cubic interactions. Figure 7 provides an illustration by showing the potential energy surfaces $V(\beta, \gamma)$ for the $\mathrm{Ru}$ isotopes obtained in the classical limit of the IBM-1 hamiltonian without and with the $\hat{B}_{3}^{\dagger} \cdot \tilde{B}_{3}$ interaction. The surfaces on the left-hand side are obtained from the two-body hamiltonian and have a prolate minimum $\left(\gamma=0^{\circ}\right)$ for $\beta \approx 1.2$. (The quadrupole deformation parameter $\beta$ is larger in the IBM than in the geometric Bohr-Mottelson model for reasons discussed in ref. [23].) The hamiltonian which includes the $\hat{B}_{3}^{\dagger} \cdot \tilde{B}_{3}$ interaction yields the surfaces on the right-hand side. One notices that in the heavier $\mathrm{Ru}$ isotopes the deformed minimum extends further towards triaxial shapes and becomes very flat in ${ }^{112} \mathrm{Ru}$ with $\beta \approx 1$ up to $\gamma \approx 15^{\circ}$. In ${ }^{112} \mathrm{Ru}$ noticeable changes of the potential $V(\beta, \gamma)$ are found as a result of the inclusion of cubic interactions which perhaps is not surprising since parameter variations are rather important between IBM-1 and IBM-1c in this nucleus (see table 3). However, even in this example with a large $v_{3}$ parameter no triaxial minimum is obtained. A similar analysis of ${ }^{114,116} \mathrm{Pd}$ yields potentials with a spherical minimum which become flatter if the $\hat{B}_{3}^{\dagger} \cdot \tilde{B}_{3}$ interaction is added to the hamiltonian.

\subsection{Xenon and barium isotopes}

Casten and von Brentano [24] pointed out the occurrence of $\mathrm{SO}(6)$-like nuclei in the region with mass number $A \sim 130$. In particular, the isotopes ${ }^{124-130} \mathrm{Xe}$ and ${ }^{128-134} \mathrm{Ba}$ were found to display properties that are consistent with the $\mathrm{SO}(6)$ limit. In a subsequent study [14] it was shown that the theoretical description of these nuclei is greatly improved via the inclusion of the threebody interaction $\hat{B}_{3}^{\dagger} \cdot \tilde{B}_{3}$. Since these early studies more members of the $\gamma$ band have been established experimentally in several Xe and $\mathrm{Ba}$ isotopes and it appears therefore worthwhile to reconsider this region as regards triaxiality features and $\gamma$-band staggering.

The fitting procedure as explained in sect. 3 leads to the parameters and rms deviations shown in table 4 . One notes that in all Xe and $\mathrm{Ba}$ isotopes the in-

clusion of the $\hat{B}_{3}^{\dagger} \cdot \tilde{B}_{3}$ interaction leads to a smaller rms deviation. Parameter fluctuations can be large, however. This is particularly the case in the Xe isotopes, with parameters in ${ }^{126} \mathrm{Xe}$ very different from those in the neighbouring 

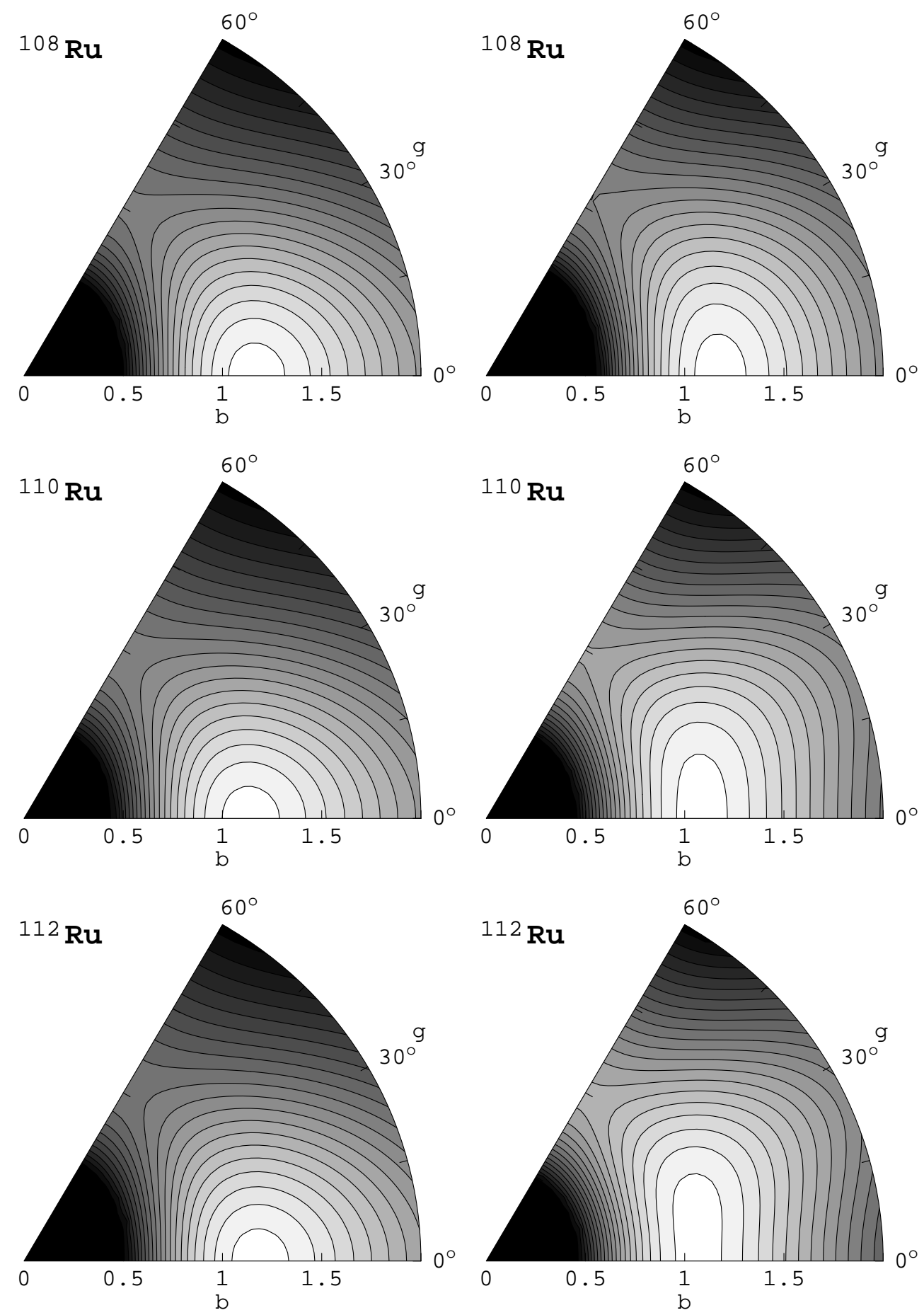

Fig. 7. Potential energy surfaces $V(\beta, \gamma)$ for the $\mathrm{Ru}$ isotopes. The plots on the left-hand side show the classical limit of the IBM-1 hamiltonian with only two-body interactions while on the right-hand side the effect of $\hat{B}_{3}^{\dagger} \cdot \tilde{B}_{3}$ is included. 
Table 4

Parameters and rms deviation for $\mathrm{Xe}$ and $\mathrm{Ba}$ isotopes in units of $\mathrm{keV}$.

\begin{tabular}{cccccccc}
\hline Nucleus & $\epsilon_{d}$ & \multicolumn{1}{c}{$\kappa$} & $\kappa^{\prime}$ & \multicolumn{1}{c}{$\lambda_{d}$} & $v_{3}$ & $\chi^{*}$ & $\sigma$ \\
\hline${ }^{124} \mathrm{Xe}$ & 922 & -65.8 & 11.3 & -145.8 & - & -0.10 & 44 \\
& 841 & -59.1 & 8.0 & -111.5 & -33.4 & -0.10 & 24 \\
${ }^{126} \mathrm{Xe}$ & 788 & -49.2 & 12.3 & -111.7 & - & -0.10 & 82 \\
& 406 & -7.4 & 6.8 & 22.7 & -105.1 & -0.10 & 18 \\
${ }^{128} \mathrm{Xe}$ & 795 & -54.0 & 11.2 & -130.0 & - & -0.10 & 42 \\
& 788 & -69.9 & 8.3 & -141.5 & -55.5 & -0.10 & 14 \\
\hline${ }^{128} \mathrm{Ba}$ & 808 & -55.8 & 7.2 & -107.7 & - & -0.10 & 77 \\
& 888 & -62.1 & 11.4 & -144.2 & 36.4 & -0.10 & 50 \\
${ }^{130} \mathrm{Ba}$ & 730 & -48.3 & 11.0 & -98.6 & - & -0.20 & 26 \\
& 828 & -51.2 & 14.4 & -130.6 & 31.1 & -0.20 & 20 \\
${ }^{132} \mathrm{Ba}$ & 700 & -23.2 & 12.6 & -70.4 & - & -0.20 & 86 \\
& 759 & -26.5 & 14.8 & -94.5 & 41.5 & -0.20 & 76 \\
\hline
\end{tabular}

*Dimensionless.

isotopes. A possible reason is the fact that the parameters in the hamiltonian are strongly correlated and hence very sensitive to slight changes in the fitted data. The results for the $\gamma$-band staggering are shown in figs. 8 and 9 . In the Xe isotopes one observes an evolution of the $\gamma$-band signature splitting with neutron number similar to the one in the Ru isotopes. The observed even-odd staggering is smaller than the one calculated without cubic interaction, and this difference increases with angular momentum $J$ to the extent that in the heavier Xe isotopes it is reversed at $J=10$. Note that the $\gamma$-band levels are known only up to $J^{\pi}=9_{\gamma}^{+}$in ${ }^{126} \mathrm{Xe}$ and up to $J^{\pi}=7{ }_{\gamma}^{+}$in ${ }^{128} \mathrm{Xe}$. It would be of interest to confirm experimentally the predicted signature splitting for the higher-spin states.

The $\gamma$ bands in the Ba isotopes are peculiar in the sense that their observed even-odd staggering is larger than the one calculated without cubic interaction. Again, this deviation increases with angular momentum $J$ and can be corrected with the $\hat{B}_{3}^{\dagger} \cdot \tilde{B}_{3}$ interaction but this time with a positive coefficient $v_{3}$ (see table 4 ). In fact, for $J \geq 8$ the signature splitting $|S(J)|$ is larger than 1 in ${ }^{128} \mathrm{Ba}$ and ${ }^{132} \mathrm{Ba}$ which corresponds to a $7_{\gamma}^{+}$above $8_{\gamma}^{+}$and a $9_{\gamma}^{+}$above $10_{\gamma}^{+}$. This peculiar behaviour can be modelled to some extent with a cubic interaction.

The positive value of the fitted coefficient $v_{3}$ has the expected effect on the 


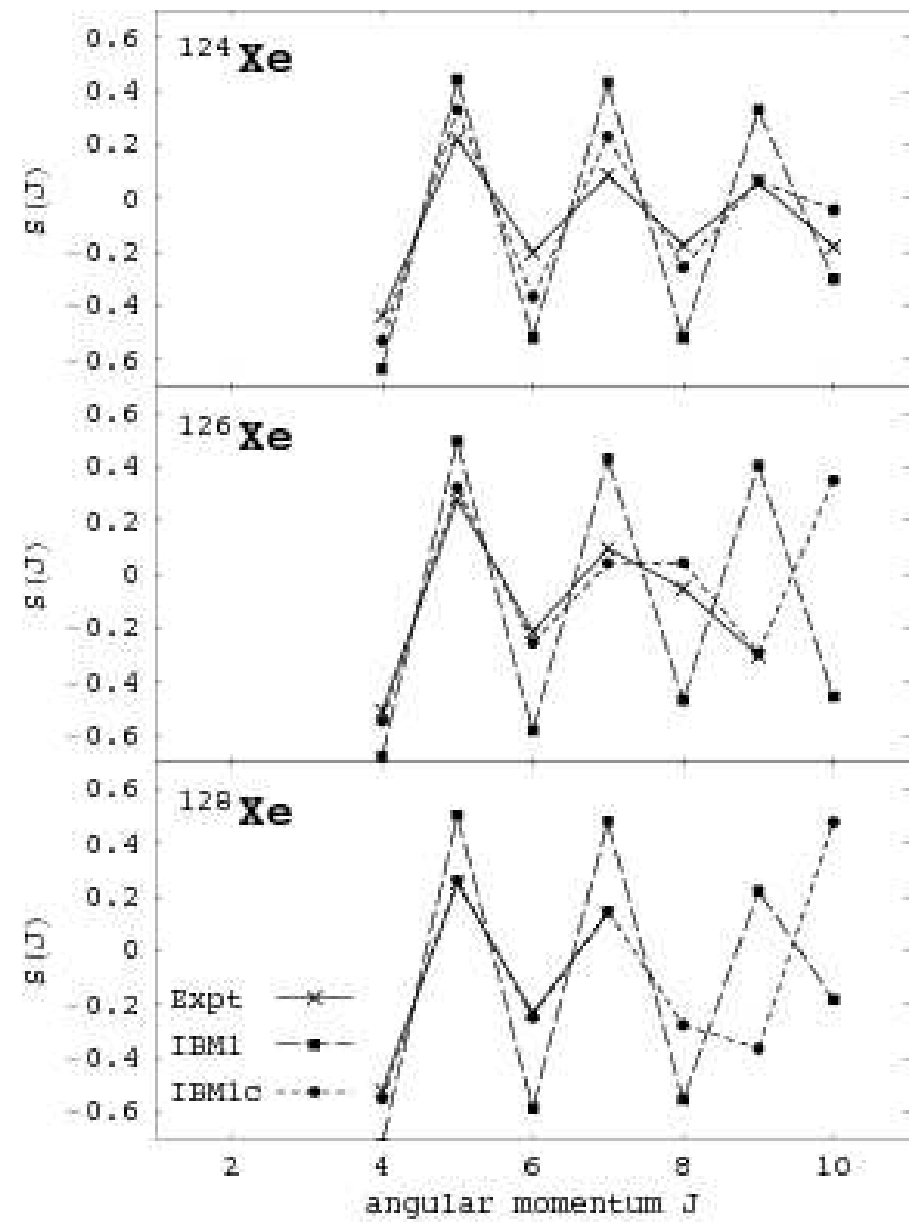

Fig. 8. Same caption as fig. 4 for the nuclei ${ }^{124-128}$ Xe.

potential $V(\beta, \gamma)$ derived in the classical limit, as is illustrated in fig. 10 for the nucleus ${ }^{128} \mathrm{Ba}$. Whereas a negative $v_{3}$ leads to a softer potential in the $\gamma$ direction (as in ${ }^{112} \mathrm{Ru}$ ), a positive value for this interaction yields a more rigid, axially symmetric shape with a slightly larger deformation $\beta$.

In the initial two-body hamiltonian the E2 transition rates depend strongly on the value of $\chi$ in the quadrupole operator. It is expected that this is still the case when cubic terms are added to the hamiltonian as long as these do not substantially alter its eigenstates. In several of the Xe and Ba isotopes many $B(\mathrm{E} 2)$ values between the low-lying states are known and these allow a test of the wave function in the cubic calculation. The results are shown in tables 5 and 6. Generally a good agreement between experimental and calculated $B(\mathrm{E} 2)$ values is obtained. One notable discrepancy is the $2_{2}^{+} \rightarrow 0_{1}^{+}$transition in ${ }^{132} \mathrm{Ba}$ with a calculated $B(\mathrm{E} 2)$ value which is an order of magnitude too small. This value is equally small in the IBM-1 calculation without cubic interaction and is due to an accidental cancellation of terms with the hamiltonian (10). 

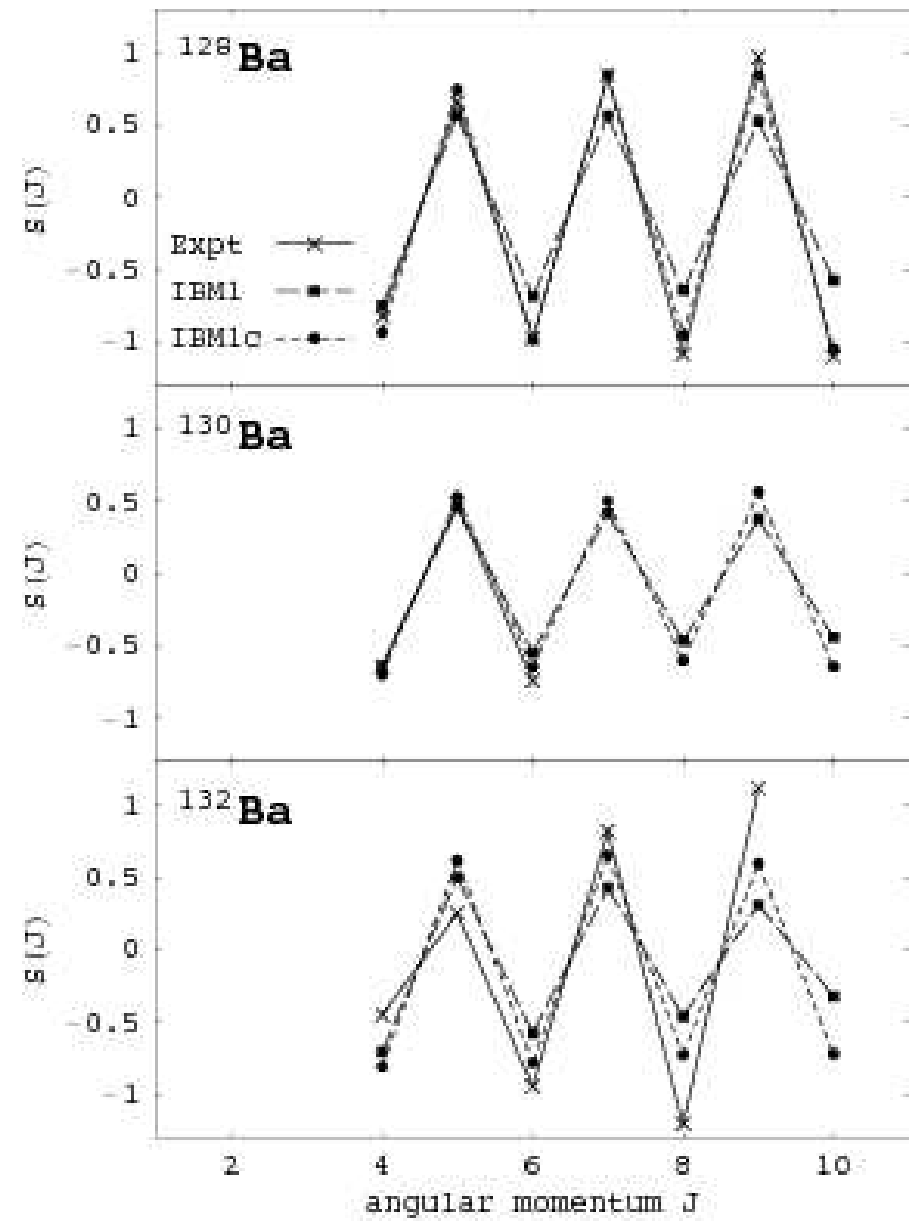

Fig. 9. Same caption as fig. 4 for the nuclei ${ }^{128-132}$ Ba.
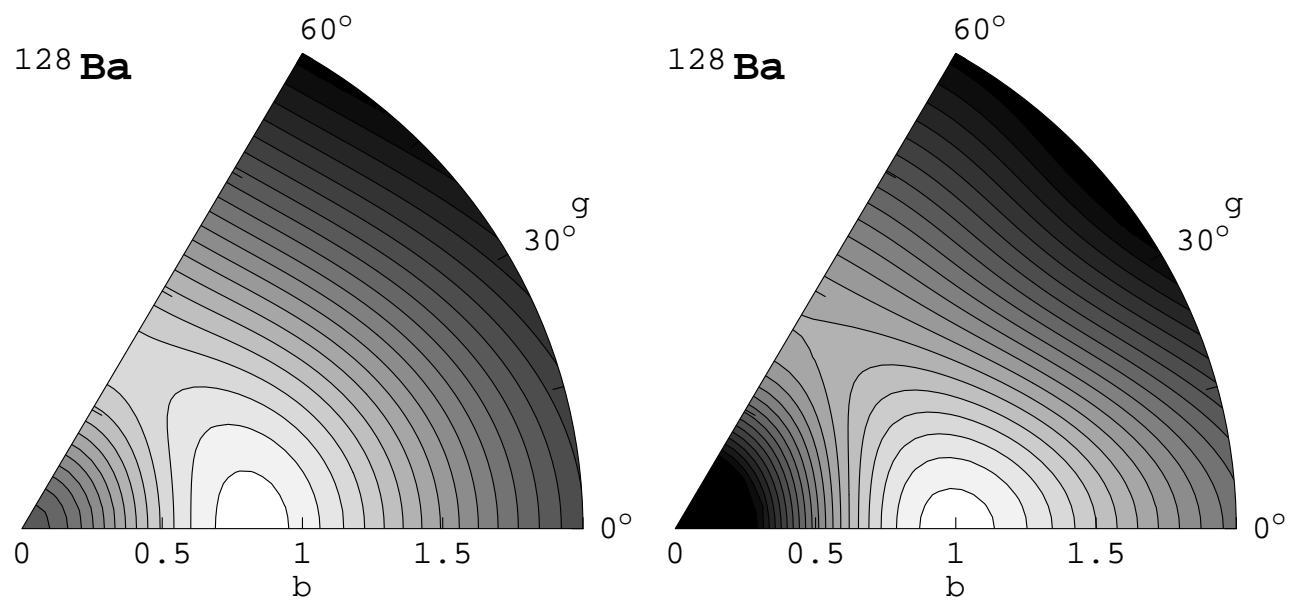

Fig. 10. Same caption as fig. 7 for the nucleus ${ }^{128}$ Ba. 
Table 5

Experimental and calculated $B(\mathrm{E} 2)$ values in units of $10^{2} \mathrm{e}^{2} \mathrm{fm}^{4}$ in ${ }^{124-128} \mathrm{Xe}$.

\begin{tabular}{|c|c|c|c|c|c|c|}
\hline \multirow[b]{2}{*}{$J_{\mathrm{i}}^{\pi} \rightarrow J_{\mathrm{f}}^{\pi}$} & \multicolumn{2}{|c|}{${ }^{124} \mathrm{Xe}$} & \multicolumn{2}{|c|}{${ }^{126} \mathrm{Xe}$} & \multicolumn{2}{|c|}{${ }^{128} \mathrm{Xe}$} \\
\hline & Expt & IBM-1c & Expt & IBM-1c & Expt & IBM-1c \\
\hline $2_{1}^{+} \rightarrow 0_{1}^{+}$ & $30(2)$ & 30 & $15(5)$ & 15 & $15(1)$ & 18 \\
\hline $4_{1}^{+} \rightarrow 2_{1}^{+}$ & $40(5)$ & 42 & - & 24 & $23(2)$ & 24 \\
\hline $6_{1}^{+} \rightarrow 4_{1}^{+}$ & $48(18)$ & 45 & - & 29 & $30(3)$ & 24 \\
\hline $8_{1}^{+} \rightarrow 6_{1}^{+}$ & $19(8)$ & 43 & - & 30 & $37(4)$ & 20 \\
\hline $2_{2}^{+} \rightarrow 0_{1}^{+}$ & $0.48(11)$ & 0.65 & - & 0.00 & $0.25(2)$ & 0.20 \\
\hline $2_{2}^{+} \rightarrow 2_{1}^{+}$ & $21(4)$ & 33 & - & 24 & $18(2)$ & 21 \\
\hline $3_{1}^{+} \rightarrow 2_{1}^{+}$ & $0.32(12)$ & 0.80 & - & 0.00 & - & 0.21 \\
\hline $3_{1}^{+} \rightarrow 2_{2}^{+}$ & $2.9(11)$ & 34 & - & 22 & - & 18 \\
\hline $4_{2}^{+} \rightarrow 2_{2}^{+}$ & $26(9)$ & 22 & - & 15 & - & 12 \\
\hline
\end{tabular}

Table 6

Experimental and calculated $B(\mathrm{E} 2)$ values in units of $10^{2} \mathrm{e}^{2} \mathrm{fm}^{4}$ in ${ }^{128-132} \mathrm{Ba}$.

\begin{tabular}{|c|c|c|c|c|c|c|}
\hline \multirow[b]{2}{*}{$J_{\mathrm{i}}^{\pi} \rightarrow J_{\mathrm{f}}^{\pi}$} & \multicolumn{2}{|c|}{${ }^{128} \mathrm{Ba}$} & \multicolumn{2}{|c|}{${ }^{130} \mathrm{Ba}$} & \multicolumn{2}{|c|}{${ }^{132} \mathrm{Ba}$} \\
\hline & Expt & IBM-1c & Expt & IBM-1c & Expt & IBM-1c \\
\hline $2_{1}^{+} \rightarrow 0_{1}^{+}$ & $28(3)$ & 29 & $22(1)$ & 22 & $17(2)$ & 26 \\
\hline $4_{1}^{+} \rightarrow 2_{1}^{+}$ & $41(2)$ & 39 & $31(1)$ & 31 & - & 40 \\
\hline $6_{1}^{+} \rightarrow 4_{1}^{+}$ & $39(3)$ & 40 & $37(2)$ & 31 & - & 44 \\
\hline $8_{1}^{+} \rightarrow 6_{1}^{+}$ & $37(5)$ & 37 & $35(1)$ & 28 & - & 39 \\
\hline $2_{2}^{+} \rightarrow 0_{1}^{+}$ & $1.3(2)$ & 1.2 & - & 1.1 & $1.5(2)$ & 0.17 \\
\hline $2_{2}^{+} \rightarrow 2_{1}^{+}$ & - & 24 & - & 17 & $57(6)$ & 37 \\
\hline $4_{2}^{+} \rightarrow 2_{1}^{+}$ & $0.34(2)$ & 0.25 & - & 0.47 & - & 0.27 \\
\hline $4_{2}^{+} \rightarrow 2_{2}^{+}$ & $24(2)$ & 24 & - & 17 & - & 23 \\
\hline $6_{2}^{+} \rightarrow 4_{1}^{+}$ & $0.30(4)$ & 0.40 & - & 0.23 & - & 0.39 \\
\hline $6_{2}^{+} \rightarrow 4_{2}^{+}$ & $38(5)$ & 28 & - & 21 & - & 27 \\
\hline
\end{tabular}

\subsection{Osmium and platinum isotopes}

Cizewski et al. [25] proposed ${ }^{196} \mathrm{Pt}$ as a first example of the $\mathrm{SO}(6)$ dynamical symmetry of the IBM-1. Subsequently, it became clear that the entire region of $\mathrm{Pt}$ and $\mathrm{Os}$ isotopes can be described as transitional between the $\mathrm{SO}(6)$ 
Table 7

Parameters and rms deviation for Os and Pt isotopes in units of keV.

\begin{tabular}{cccccccc}
\hline Nucleus & $\epsilon_{d}$ & $\kappa$ & $\kappa^{\prime}$ & $\lambda_{d}$ & $v_{3}$ & $\chi^{*}$ & $\sigma$ \\
\hline${ }^{180}$ Os & 946 & -29.1 & 4.1 & -62.5 & - & -0.30 & 19 \\
& 634 & -41.2 & -5.1 & -12.0 & -58.7 & -0.30 & 10 \\
\multirow{2}{*}{${ }^{182}$ Os } & 1013 & -32.7 & 5.7 & -81.3 & - & -0.30 & 8 \\
& 813 & -37.8 & 2.8 & -62.3 & -14.9 & -0.30 & 9 \\
${ }^{184} \mathrm{Os}$ & 844 & -40.6 & 6.8 & -89.7 & - & -0.30 & 2 \\
& 973 & -35.6 & 9.3 & -99.2 & 10.0 & -0.30 & 1 \\
${ }^{186} \mathrm{Os}$ & 871 & -34.8 & 10.9 & -95.7 & - & -0.30 & 5 \\
& 752 & -40.4 & 7.8 & -84.5 & -16.1 & -0.30 & 1 \\
\hline${ }^{186} \mathrm{Pt}$ & 747 & -33.7 & 3.7 & -65.9 & - & -0.20 & 36 \\
& 449 & -15.2 & -3.9 & 15.5 & -60.5 & -0.20 & 24 \\
\hline
\end{tabular}

${ }^{*}$ Dimensionless.

and $\mathrm{SU}(3)$ limits [26]. In this subsection we present a detailed analysis of Os and Pt isotopes of which enough $\gamma$-band levels are firmly established for the staggering analysis to be meaningful. In both ${ }^{186} \mathrm{Os}$ and ${ }^{186} \mathrm{Pt}$ the $\gamma$-band levels are known up $J^{\pi}=10^{+}$such that its staggering properties can be fitted reliably in these nuclei. Less information is available in ${ }^{180} \mathrm{Os}$ and ${ }^{182} \mathrm{Os}$ (up to $7_{\gamma}^{+}$), and even less in ${ }^{184}$ Os (up to $6{ }_{\gamma}^{+}$). The latter nucleus, although relatively poorly known, is included in the analysis since it is intermediate between two better known isotopes.

The parameters resulting from the fits are shown in table 7. Since the Os isotopes are further removed from $\mathrm{SO}(6)$ and closer to $\mathrm{SU}(3)$, the value of $|\chi|$ is larger than in the other nuclei considered so far. The more deformed character of the Os isotopes also leads to a parameter systematics which is smoother than in previous examples. It should be noted, however, that in ${ }^{184}$ Os the sign of $v_{3}$ comes out positive while in the other isotopes it is negative. This conceivably might be due to the fact that not enough levels are known in the $\gamma$ band of this nucleus. The main outcome of the fits to ${ }^{184} \mathrm{Os}$ and ${ }^{186} \mathrm{Os}$ is that the $\hat{B}_{3}^{\dagger} \cdot \tilde{B}_{3}$ interaction is small. The results for the $\gamma$-band staggering in ${ }^{180-186} \mathrm{Os}$ and in ${ }^{186} \mathrm{Pt}$ are shown in figs. 11 and 12, respectively. Given the low rms deviations obtained in the fit, it comes as no surprise that in all nuclei the observed signature splitting of the $\gamma$ band is accurately reproduced. It is also seen that the inclusion of the $\hat{B}_{3}^{\dagger} \cdot \tilde{B}_{3}$ interaction leads to a systematic improvement in the description of the $\gamma$ band. A noteworthy example is ${ }^{186}$ Os. The standard two-body IBM-1 hamiltonian already gives an excellent description with an rms deviation of only $\sigma=5 \mathrm{keV}$. Nevertheless, there is a small but consistent 

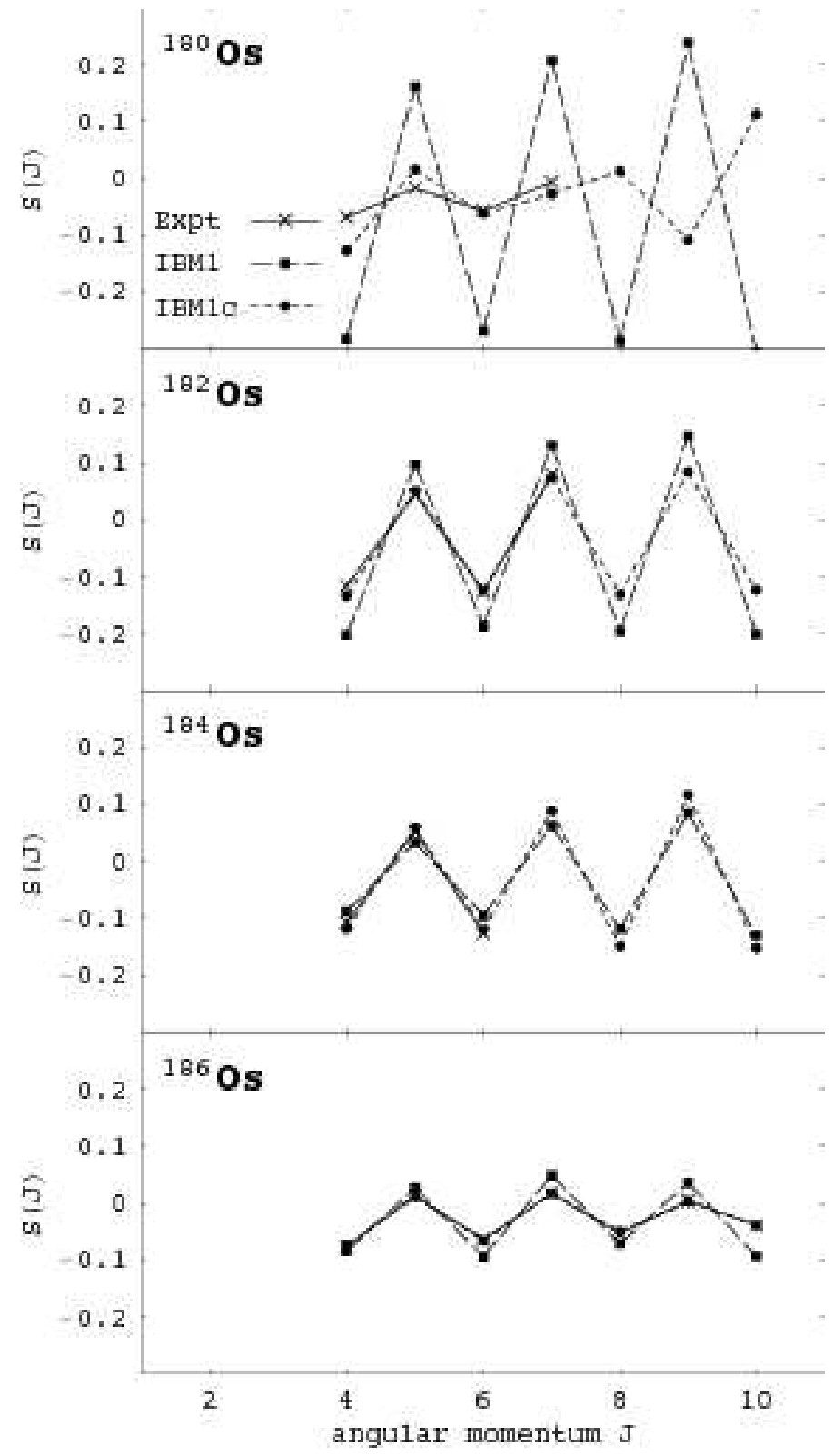

Fig. 11. Same caption as fig. 4 for the nuclei ${ }^{180-186}$ Os.

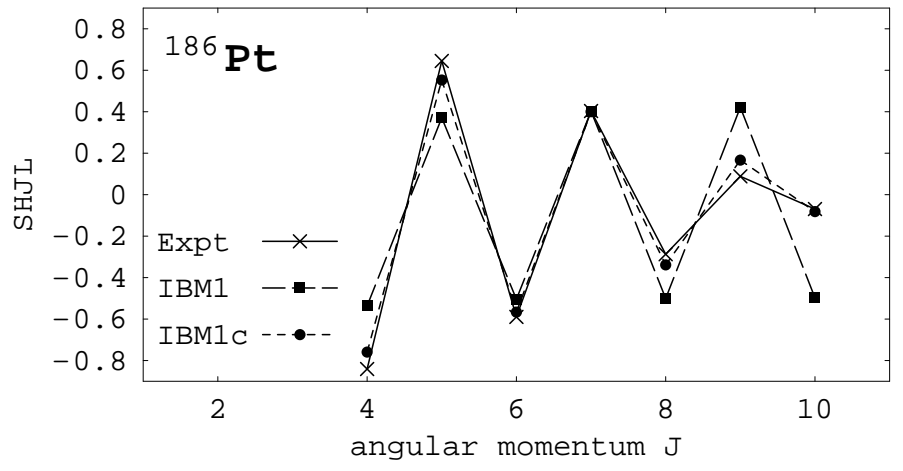

Fig. 12. Same caption as fig. 4 for the nucleus ${ }^{186} \mathrm{Pt}$. 
Table 8

Experimental and calculated $B(\mathrm{E} 2)$ values in units of $10^{2} \mathrm{e}^{2} \mathrm{fm}^{4}$ in ${ }^{180-186}$ Os.

\begin{tabular}{|c|c|c|c|c|c|c|}
\hline \multirow[b]{2}{*}{$J_{\mathrm{i}}^{\pi} \rightarrow J_{\mathrm{f}}^{\pi}$} & \multicolumn{2}{|c|}{${ }^{180} \mathrm{Os}$} & \multirow{2}{*}{$\frac{{ }^{182} \mathrm{Os}}{\mathrm{IBM}-1 \mathrm{c}}$} & \multirow{2}{*}{$\frac{{ }^{184} \mathrm{Os}}{\mathrm{IBM}-1 \mathrm{c}}$} & \multicolumn{2}{|c|}{${ }^{186} \mathrm{Os}$} \\
\hline & Expt & IBM-1c & & & Expt & IBM-1c \\
\hline $2_{1}^{+} \rightarrow 0_{1}^{+}$ & $76(19)$ & 74 & 77 & 61 & $58(2)$ & 69 \\
\hline $4_{1}^{+} \rightarrow 2_{1}^{+}$ & $122(16)$ & 107 & 110 & 86 & $85(4)$ & 97 \\
\hline $6_{1}^{+} \rightarrow 4_{1}^{+}$ & $101(25)$ & 119 & 120 & 90 & $116(3)$ & 104 \\
\hline $8_{1}^{+} \rightarrow 6_{1}^{+}$ & $40(8)$ & 125 & 124 & 88 & $110(6)$ & 103 \\
\hline $2_{2}^{+} \rightarrow 0_{1}^{+}$ & - & 4.3 & 3.9 & 2.4 & $6.4(3)$ & 3.1 \\
\hline $2_{2}^{+} \rightarrow 2_{1}^{+}$ & - & 17 & 11 & 4.6 & $14.8(5)$ & 8.6 \\
\hline $2_{2}^{+} \rightarrow 4_{1}^{+}$ & - & 1.3 & 0.72 & 0.32 & $0.8(3)$ & 0.60 \\
\hline $4_{2}^{+} \rightarrow 2_{1}^{+}$ & - & 0.37 & 0.80 & 1.0 & $2.0(2)$ & 0.70 \\
\hline $4_{2}^{+} \rightarrow 4_{1}^{+}$ & - & 17 & 11 & 4.2 & $15.6(13)$ & 8.3 \\
\hline $4_{2}^{+} \rightarrow 2_{2}^{+}$ & - & 41 & 42 & 33.0 & $45(4)$ & 34 \\
\hline $6_{2}^{+} \rightarrow 4_{1}^{+}$ & - & 0.00 & 0.26 & 0.79 & $0.80(7)$ & 0.24 \\
\hline $6_{2}^{+} \rightarrow 4_{2}^{+}$ & - & 77 & 79 & 60 & $75(7)$ & 62 \\
\hline
\end{tabular}

deviation in the staggering pattern of the $\gamma$ band which is remedied with the cubic term. The nucleus ${ }^{186} \mathrm{Os}$ is also well studied as regards its E2 decay properties and, as can be seen from table 8, all its known $B(\mathrm{E} 2)$ values are rather well reproduced by the calculation. In two of the four isotopes, namely ${ }^{182} \mathrm{Os}$ and ${ }^{184} \mathrm{Os}$, only one or two $B(\mathrm{E} 2)$ values are known experimentally, and for these nuclei only theoretical predictions are quoted in the table.

As a final outcome of this study we show in fig. 13 the potential energy surfaces for the different Os isotopes which are obtained in the classical limit of the IBM-1 hamiltonian with cubic interactions. It shows the evolution with increasing mass number $A$ towards a potential with a more deformed minimum and which is more rigid in $\gamma$.

\section{Conclusions}

Two main conclusions can be drawn from this work. First, very accurate nucleus-by-nucleus fits can be achieved with the simplified IBM-1 hamiltonian (10) to which a single three-body interaction of the type (12) is added. In almost all $\gamma$-soft nuclei studied the inclusion of the $\hat{B}_{3}^{\dagger} \cdot \tilde{B}_{3}$ interaction yields a consistently better description of the signature splitting of the $\gamma$ band. This 

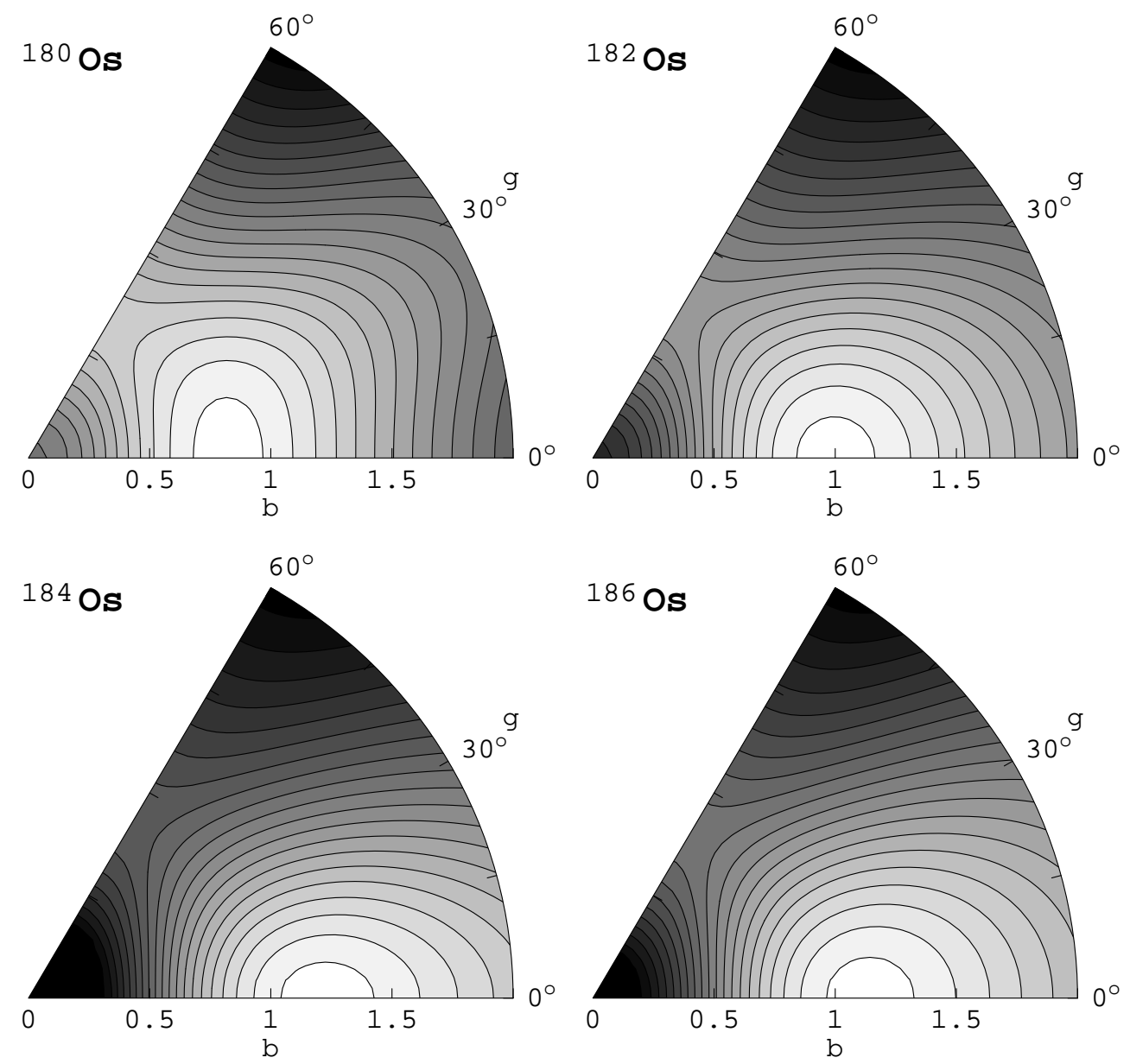

Fig. 13. Potential energy surfaces $V(\beta, \gamma)$ for the nuclei ${ }^{180-186}$ Os. All plots show the classical limit of the IBM-1 hamiltonian with the cubic interaction included.

improved description of the $\gamma$-band energies is obtained while maintaining good agreement for the E2-decay properties. Nevertheless, no systematics of three-body parameters could be established. While we have currently a good working hamiltonian which includes up to two-body interactions and which describes nuclei throughout the nuclear chart, little is known of the overall trends for three-body interactions.

A second conclusion concerns the geometry underlying the algebraic hamiltonians, as was illustrated with several examples. One surprising outcome of our approach is that an unbiased fit of energy levels in some nuclei $\left({ }^{114,116} \mathrm{Pd}\right.$, ${ }^{126} \mathrm{Xe},{ }^{132} \mathrm{Ba}$ and ${ }^{186} \mathrm{Pt}$ ) leads to an IBM-1 hamiltonian of which the classical limit yields a potential with a spherical minimum whereas these nuclei usually are considered $\gamma$ soft and weakly deformed. The additional three-body interaction $v_{3} \hat{B}_{3}^{\dagger} \cdot \tilde{B}_{3}$, introduced to improve the description of the $\gamma$ band, makes the potential energy surface $V(\beta, \gamma)$ softer in $\gamma$ for $v_{3}<0$ and more rigid for $v_{3}>0$. The sign of this coefficient follows from the staggering pattern of the $\gamma$ band. Finally, in none of the nuclei studied we found evidence for a triaxial 
minimum.

\section{Acknowledgement}

We wish to thank Adrian Gelberg for his valuable comments on this work.

\section{References}

[1] A. Bohr and B.R. Mottelson, Mat. Fys. Medd. Dan. Vid. Selsk. 27, No 16 (1953).

[2] F. Iachello and A. Arima, The Interacting Boson Model (Cambridge University Press, Cambridge, 1987).

[3] J.N. Ginocchio and M.W. Kirson, Phys. Rev. Lett. 44 (1980) 1744.

[4] A.E.L. Dieperink, O. Scholten and F. Iachello, Phys. Rev. Lett. 44 (1980) 1747.

[5] A. Bohr and B.R. Mottelson, Phys. Scripta 22 (1980) 468.

[6] A. Bohr and B.R. Mottelson, Nuclear Structure. II Nuclear Deformations (Benjamin, Reading, Massachusetts, 1975).

[7] P. Van Isacker and J.-Q. Chen, Phys. Rev. C 24 (1981) 684.

[8] D.D. Warner and R.F. Casten, Phys. Rev. Lett. 48 (1982) 1385.

[9] P.O. Lipas, P. Toivonen and D.D. Warner, Phys. Lett. B 155 (1985) 295.

[10] X.-W. Pan, T. Otsuka, J.-Q. Chen and A. Arima, Phys. Lett. B 287 (1992) 1.

[11] A. Arima and F. Iachello, Ann. Phys. (NY) 123 (1979) 468.

[12] A.S. Davydov and G.F. Filippov, Nucl. Phys. 8 (1958) 237.

[13] K. Heyde, P. Van Isacker, M. Waroquier and J. Moreau, Phys. Rev. C 29 (1984) 1420.

[14] R.F. Casten, P. von Brentano, K. Heyde, P. Van Isacker and J. Jolie, Nucl. Phys. A 349 (1985) 289.

[15] A. Arima and F. Iachello, Ann. Phys. (NY) 111 (1978) 201.

[16] Y. Alhassid and A. Leviatan, J. Phys. A 25 (1992) L1265.

[17] A. Leviatan, Phys. Rev. Lett. 77 (1996) 818.

[18] N.V. Zamfir and R.F. Casten, Phys. Lett. B 260 (1991) 265. 
[19] National Nuclear Data Center, http://www.nndc.bnl.gov.

[20] J. Stachel, P. Van Isacker and K. Heyde, Phys. Rev. C 25 (1982) 650.

[21] P.M. Gore et al., Eur. Phys. J. A 25, s01 (2005) 471.

[22] I. Stefanescu et al., Nucl. Phys. A 789 (2007) 125.

[23] J.N. Ginocchio and M.W. Kirson, Nucl. Phys. A 350 (1980) 31.

[24] R.F. Casten and P. von Brentano, Phys. Lett. B 152 (1985) 22.

[25] J.A. Cizewski, R.F. Casten, G.J. Smith, M.L. Stelts, W.R. Kane, H.G. Börner and W.F. Davidson, Phys. Rev. Lett. 40 (1978) 167.

[26] R.F. Casten and J.A. Cizewski, Phys. Lett. B 79 (1978) 5. 